\title{
Prevalence of Dentine Hypersensitivity in a General Dental Practice in Greece.
}

\author{
N.A. Chrysanthakopoulos ${ }^{1}$ \\ ${ }^{1}$ Dental Surgeon DDSc. Resident in Maxillofacial and Oral Surgery, 401 General Military. Hospital of Athens, Athens
}

Correspondence:

35, Zaimi Street

P.C. 26223

Patra, Greece

Email:nikolaos_c@hotmail.com

\author{
Chrysanthakopoulos NA. Prevalence of Dentine Hypersensitivity in a \\ General Dental Practice in Greece. J Clin Exp Dent. 2011;3(5):e445-51. \\ http://www.medicinaoral.com/odo/volumenes/v3i5/jcedv3i5p445.pdf

\begin{abstract}
Objectives: The aim of this study was to estimate the prevalence of dentine hypersensitivity in a sample of patients visiting a general dental practice.

Study design: The study population consisted of 1,450 patients, 690 males and 760 females, aged 18-69 years. All individuals were clinically examined and answered questions regarding variables such as gender, age, educational level, occupation status, teeth affected and any factor that initiated the sensitivity. The clinical examination involved assessment of sensitive teeth per patient, while the amount of buccal gingival recession associated with the sensitive teeth was also recorded. Statistical analysis performed by using methods of descriptive statistics and chi square-test.

Results: Two hundred and sixty four patients were diagnose as having dentine hypersensitivity, giving a prevalence rate $18.2 \%$. Prevalence rate for hypersensitivity in females $(19.34 \%)$ was significantly higher $(\mathrm{p}=0.0015)$ than males $(16.95 \%)$. The mean number of sensitive teeth per patient showed a peak in the 40-49 year age group in males and in 60-69 year age group in females. The commonest teeth affected by dentine hypersensitivity were the first and second premolars, the canines and the first molars of maxilla and mandible. The majority (85.9\%) of sensitive teeth had at least 1-3 $\mathrm{mm}$ of gingival recession while the most commonest pain-initiating stimuli was the consumption of cold drinks (56.1\%). A statistically significant difference recorded between dentine hypersensitivity and educational level $(\mathrm{p}=0.00094)$.

Conclusions: The prevalence of dentine hypersensitivity in the adult sample of the present study was $18.5 \%$. There was also a tendency for the patients with sensitive teeth to come from higher social classes.
\end{abstract}

Key words: prevalence, dentine hypersensitivity, gingival recession, epidemiology 


\section{Introduction}

Dentine hypersensitivity (DH) has been defined as a "short, sharp pain arising from exposed dentine response to stimuli typically thermal, evaporative, tactile, osmotic or chemical and which cannot be ascribed to any other form of dental defect or disease" (1-3).

DH is a common condition that is frequently encountered in dental practice. Several studies have reported that DH was found in adult populations, with prevalence ranging from $1.34 \%$ to $74 \%(4-22)$.

This wide variation in prevalence has been presumed to be due to a number of factors, including different methods used to diagnose the condition (clinical examination, questionnaire, etc.), variation in the consumption of erosive drinks, variation in the type of sample population and the type of setting where the study was carried out (3).

Limited data have been collected previously from questionnaires studies rather than by clinical investigation (10).

Most previous studies were mainly carried out in university hospitals or dental practices $(3-8,12,16,18,19)$, but these selected sample populations could experience more dental or periodontal diseases than in the general population. Many people with minor tooth sensitivity do not necessarily seek professional advice or dental treatment as well, making it more difficult to obtain an accurate prevalence of DH for the general population than for those in hospitals or clinics.

It has been shown that the aetiology of DH is multi-factorial, however interactions between several factors including stimuli as well as predisposing factors may play an important role in initiating this condition (13, 23-24). Cold and air stimulation are known to be the commonest stimuli $(15,25)$ while dietary acid is also shown to have a significant potential in evoking DH (18). Among the predisposing factors for $\mathrm{DH}$, gingival recession and abrasion, as well as erosion and attrition have been considered as important ones (23-24).

Especially, gingival recession can result in exposure of the root surfaces and has been considered a common risk factor or contributing feature for subsequent $\mathrm{DH}$, and previous studies have reported prevalence of DH associated with gingival recession ranging from $29.7 \%$ to 93\% (3-6, 9).

$\mathrm{DH}$ is also a common finding in patients with chronic periodontal disease since the root surface may be exposed as part of the disease process. DH prevalence is higher in this group of patients and ranging from $72.5 \%$ to $98 \%(6,9,26)$. Based on these observations Dababneh et al. (26) suggested that the DH associated with periodontal disease may have a different aetiology, possibly related to bacterial penetration of the dentinal tubules. As a result of this possibility, the European Federation of Periodontology (EFP) have recommended the use of the term root sensitivity (RS) to describe the sensitivity associated with periodontal diseases and treatments (5). Similar studies have not been carried out in Greece, therefore it is important to collect detailed information from questionnaire and clinical examination of this condition of subjects who attended a private practice in order to assess the epidemiology of DH.

The present cross-sectional study was designed to estimate the prevalence of DH in an adult population sample in Greece.

\section{Material and Methods}

Subject population

Study population consisted of 1,450 subjects, 690 males and 760 females, 18-69 years of age (mean age $47.6 \pm$ 3.7 years) of which 1,024 attended a private dental practice and 426 selected randomly as they visited the above mentioned practice for their dental follow-up which is organized by the Greek Ministry of Health annually. Subjects with less than 24 teeth or those who had undergone periodontal therapy or were on antibiotic or antiinflammatory therapy within the past six months were excluded from the study which ran from October 2010 to March 2011 and all examinations were performed by one dentist. The participants were in good general health as estimated by a health questionnaire.

The study population divided into 6 groups according to the age range: group I-18 to 19 years: 214 subjects (104 males, 110 females); group II-20 to 29 years: 248 subjects (116 males, 132 females); group III-30 to 39 years: 236 subjects (112 males, 124 females); group IV-40 to 49 years: 258 subjects (123 males, 135 females); group V-50 to 59 years: 244 subjects (120 males, 124 females) and group VI-60 to 69 years: 250 subjects (115 males, 135 females).

\section{Ethical considerations}

The present study was not an experimental one. In Greece only experimental studies must be reviewed and approved by authorized committees (Dental Schools, Greek Dental Associations, Ministry of Health, etc.)

Subjects who agreed to participate in the present study informed about the evaluation to which they would be submitted and signed an informed consent form.

\section{Inclusion criteria}

The selection criteria of the participants comprised age above 18 and up to 69 years and a mean number of 20 natural teeth, since large numbers of missing teeth might interfere with the results of the present study. More than 12 missing teeth can cause problems with eating, speech, and other basic activities and could lead to over- or underestimate the prevalence of $\mathrm{DH}$.

\section{Questionnaire}

Before the clinical examination all subjects filled in a questionnaire regarding data such as gender, age, educational level (primary, secondary, college, universi- 
ty), occupation status, teeth affected by hypersensitivity, any factor that initiated the sensitivity (cold/hot drinks, cold/hot food, sour stimuli, tooth brushing, sweet food) and the last visit to the dentist.

The relationship between DH and social class which determined by the occupation status was examined using the Registrar General's Classification of Occupations as used by Bradnock et al. (27) in the UK adult dental health survey. This divides occupations into a series of six classes using the following criteria: class I Professional (e.g., doctor, dentist, lawyer), class II Managerial and lower professional (e.g., manager, nurse, school teachers), class III N Skilled, non-manual (e.g., clerk, cashier), class III M Skilled, manual (e.g., carpenter, bricklayer, coal face worker), class IV Semi-skilled, manual (e.g., postman, agriculture worker) and class V Unskilled manual (porter, ticket collector, general labourer).

\section{Clinical Examination}

In order to make the diagnosis of $\mathrm{DH}$, other condition or pathology such as dental caries must be rejected. The diagnosis of DH was made as the result of both clinical examination and patient's indication. Initially, the teeth and gingiva were dried with compressed air gently and patients were asked if they had any sensitivity. An observation of dentin hypersensitivity was made considering both patient's indication of the problems well as clinical findings.

In case the response was positive the diagnosis of $\mathrm{DH}$ was confirmed using a blast of air from a syringe of dental unit. In cases the response was doubtful a piece of cotton impregnated in cold water was used to confirm the definitive diagnosis of DH. In order to estimate the apico-coronal width of recession, linear measurements of gingival recession were obtained from the cemento-enamel junction up to the gingival margin in teeth presenting with gingival recession. A William's $\mathrm{mm}$ probe (PCP 10-SE, Hu-Friedy Mfg. Co. Inc., Chicago, IL, USA) was used for the purpose and the mid-facial buccal surfaces of all teeth were examined except for the third molars.

Teeth with cervical carious or wear lesions were included in the study in order to estimate the apico-coronal width of recession but were excluded from the assessment of DH prevalence, as all teeth with carious or wear lesions at any tooth surface.

Reproducibility

A randomly chosen sample of $290(20 \%)$ individuals was re-examined clinically by the same dentist in order to establish the intra-examiner variance. After consideration of the code numbers of the double examined participants no differences were recorded between the $1^{\text {st }}$ and the $2^{\text {nd }}$ clinical assessment. It is obvious that in this case the intra-examiner consistency of clinical recordings per

\begin{tabular}{|c|c|c|c|c|c|c|c|}
\hline \multirow{2}{*}{$\begin{array}{c}\text { Age } \\
\text { group }\end{array}$} & Total & Males & Females & Total & Males & Females & $\begin{array}{c}\text { M:F ratio } \\
\text { (for patients } \\
\text { with sensiti- } \\
\text { ve teeth) }\end{array}$ \\
\hline $18-19$ & 214 & 104 & 110 & 32 & 15 & 17 & $1: 1.13$ \\
\hline $20-29$ & 248 & 116 & 132 & 39 & 18 & 21 & $1: 1.16$ \\
\hline $30-39$ & 236 & 112 & 124 & 47 & 21 & 26 & $1: 1.24$ \\
\hline $40-49$ & 258 & 123 & 135 & 53 & 23 & 30 & $1: 1.3$ \\
\hline $50-59$ & 244 & 120 & 124 & 62 & 27 & 35 & $1: 1.3$ \\
\hline $60-69$ & 250 & 115 & 135 & 31 & 13 & 18 & $1: 1.38$ \\
\hline Total & 1,450 & 690 & 760 & 264 & 117 & 147 & $1: 1.25$ \\
\hline
\end{tabular}

Table 1. Distribution of the sample of the study by gender, age group and M/F ratio

\begin{tabular}{|c|c|c|c|c|c|c|}
\hline \multirow{2}{*}{$\begin{array}{c}\text { Age group } \\
\text { (years old) }\end{array}$} & \multicolumn{3}{|c|}{ Prevalence of DH (\%) } & \multicolumn{3}{c|}{ Mean number of sensitive teeth per patient } \\
\cline { 2 - 7 } & Males & Females & Total & Males & Females & Total \\
\hline $18-19$ & 14.42 & 15.45 & 14.95 & 4.13 & 4.53 & 4.34 \\
\hline $20-29$ & 15.51 & 15.90 & 15.72 & 4.33 & 4.04 & 4.18 \\
\hline $30-39$ & 18.75 & 20.96 & 19.91 & 4.67 & 4.34 & 4.49 \\
\hline $40-49$ & 18.70 & 22.20 & 20.54 & 5.10 & 3.96 & 4.45 \\
\hline $50-59$ & 22.50 & 28.22 & 25.41 & 4.74 & 3.48 & 4.03 \\
\hline $60-69$ & 11.30 & 13.30 & 12.40 & 5.77 & 4.88 & 5.25 \\
\hline
\end{tabular}

Table 2. Prevalence of dentine hypersensitivity by gender and age group and mean number of sensitive teeth per patient by gender and age group 
sextant was not estimated by $\mathrm{k}$ index.

Statistical Analysis

The individual was the statistical unit in order to estimate the prevalence of DH.

Methods of descriptive statistics and $\mathrm{x}^{2}$ test were employed to analyse the data and to confirm or not whether exists any difference between categorical variables of the present study. The data analysis was performed using the statistical package of SPSS ver.17.0 (SPSS Inc., Chicago, IL, USA). A p value less than 5\% was considered to be statistically significant.

\section{Results}

A total of 1,162 teeth were diagnosed as having DH in 264 patients giving an overall prevalence value of $18.2 \%$ $(16.95 \%$ in males and $19.34 \%$ in females, difference statistically significant, $p=0.0015)$. One hundred and seventeen subjects with DH were males (44.32\%) and 147 females $(55.68 \%)$ giving an overall male to female ratio $1: 1.25(\mathrm{p}=0.0052)$.

Table 1 presents the distribution of patients by gender/ age group and male/female ratio by gender/age group in subjects with DH.

The prevalence of DH by gender and age group and the

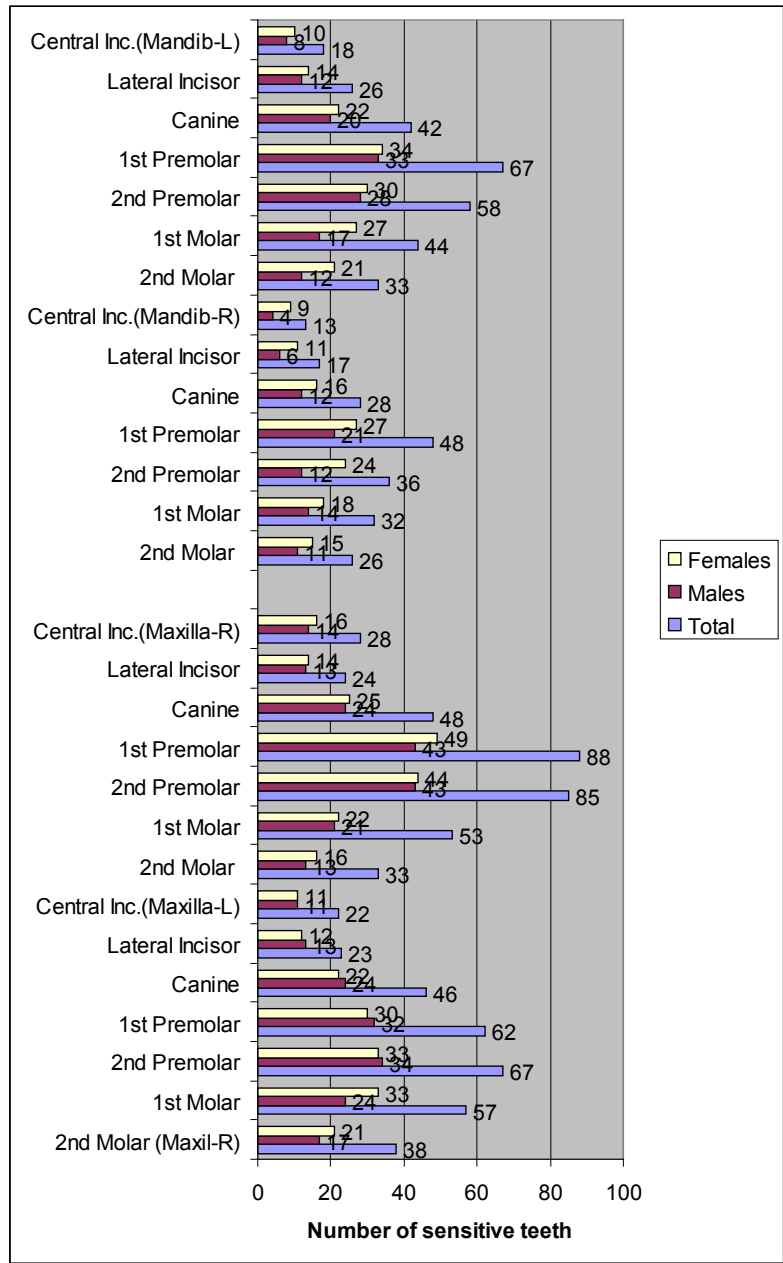

Fig. 1. Dentine hypersensitivity by tooth type in maxilla and mandible mean number of sensitive teeth per patient is shown in table 2 .

Figure 1 shows the distribution of DH by tooth type in maxilla and mandible.

Figure 2 presents the distribution of gingival recession associated with sensitive teeth by gender. The majority $(85.9 \%)$ of sensitive teeth had at least $1-3 \mathrm{~mm}$ of gingival recession.

Initiating factors of $\mathrm{DH}$ are shown in Figure 3. The most common stimuli was the consumption of cold drinks for both genders.

The distribution of patients with sensitive teeth according to educational level of the

sample is shown in table 3 .

The distribution of patients with sensitive teeth according to social class was the following: class I: $68(25.75 \%)$, class II: 83 (31.44\%), class III N: $44(16.67 \%)$, class III M: $27(10.23 \%)$, class IV: $12(4.54 \%)$ and class

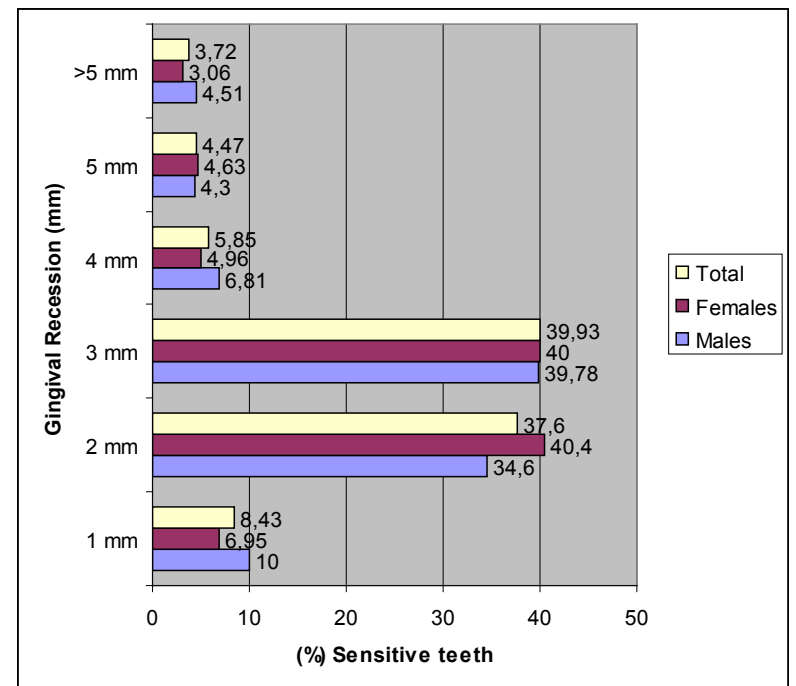

Fig. 2. Gingival recession associated with sensitive teeth

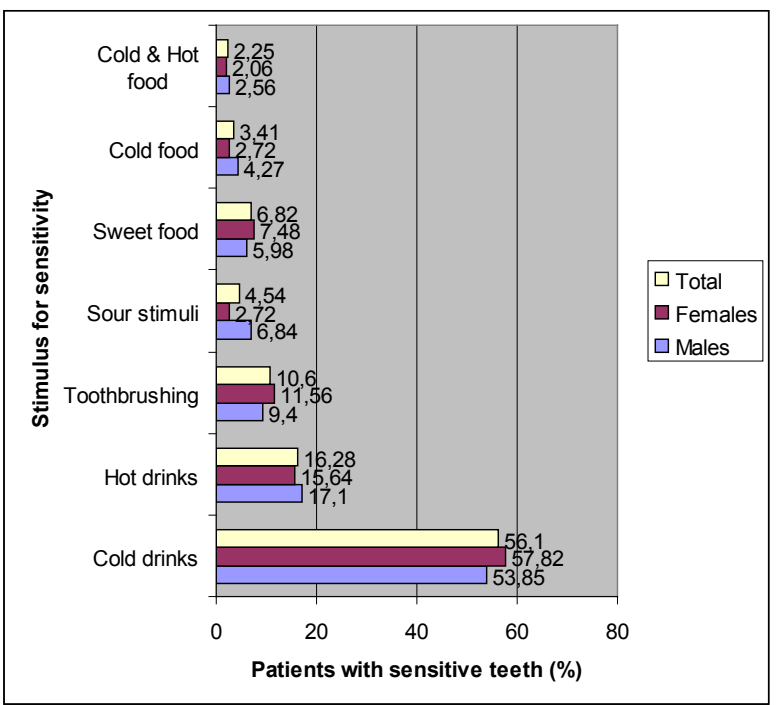

Fig. 3. Distribution of pain-initiating stimulus e448 


\begin{tabular}{|l|c|c|}
\hline & $\begin{array}{c}\text { Subjects with } \\
\text { DH }\end{array}$ & $\begin{array}{c}\text { Subjects wi- } \\
\text { thout DH }\end{array}$ \\
\hline Primary Education & $84^{*}$ & 273 \\
\hline Secondary Education & 43 & 305 \\
\hline College & 45 & 298 \\
\hline University & $92^{*}$ & 310 \\
\hline TOTAL & 264 & 1,186 \\
\hline
\end{tabular}

* P: 0.00094

Table 3. Distribution of subjects with and without sensitive teeth according to their educational level

V: $30(11.37 \%)$ (difference statistically significant, $p$ : $0.00345)$.

Table 4 presents DH by age, gender and tooth affected (jaw/position) and 95\% CI (confidence interval).

\section{Discussion}

According to the results of the questionnaire and clinical examination the present study showed that the prevalence of DH was overall $18.2 \%$. This prevalence was somewhat different from other studies conducted in dental practices and reported varying prevalence values from $2.8 \%$ to $57.2 \%(3-5,10,12,17,19)$.

Studies conducted in university clinics in which the diagnosis of DH was confirmed using clinical examination, reported higher prevalence values of $>30 \%$ $(4,6,7,9,11,16,18)$. This may be a reflection of the smaller sample sizes and sample population from the periodontology departments at these universities.

The wide variation in prevalence of $\mathrm{DH}$ could be attributed to several factors. Some of those studies have used questionnaires without concomitant clinical examinations to assess prevalence of DH. This methodology is likely to overestimate the prevalence of $\mathrm{DH}$ as the sensitivity recorded could be attributed to other factors such as dental caries. In addition most subjects investigated in studies that conducted in dental practices were below 50 years of age.

In the present study the diagnosis of DH was confirmed based on the positive response of the patients followed by clinical examination in which an air blast from a dental air syringe was used as a stimulus test (and a piece of cotton impregnated in cold water, in difficult cases, as mentioned above). Previous studies used only an air blast stimulus to clinically diagnose DH $(3,4-7)$ while other studies used evaporative and tactile stimuli in which Liu et al.(11) reported that $92 \%$ of sensitive subjects were sensitive to an air blast stimulus.

It is obvious that the results of the present study are not comparable to those of previous studies because the diagnosis of DH was confirmed using different methodology. However, the above observations highlight the main reason for the wide prevalence of $\mathrm{DH}$.

It is believed that $\mathrm{DH}$ occurs more frequently in fema-

\begin{tabular}{|c|c|c|c|c|}
\hline & \multirow{2}{*}{$\mathbf{n}$} & \multirow{2}{*}{$(\%)$} & \multicolumn{2}{|c|}{ Extent of DH } \\
\hline & & & Mean & $95 \% \mathrm{CI}$ \\
\hline \multicolumn{5}{|l|}{ Age } \\
\hline$<50$ & 171 & 64.77 & 4.38 & $4.22-4.54$ \\
\hline$>50$ & 93 & 35.23 & 4.44 & $4.23-4.65$ \\
\hline \multicolumn{5}{|l|}{ Gender } \\
\hline Males & 117 & 44.32 & $4.77^{*}$ & $4.55-4.95$ \\
\hline Females & 147 & 55.68 & $4.11 *$ & $3.93-4.29$ \\
\hline \multicolumn{5}{|c|}{ Tooth affected (position) } \\
\hline Posterior & 162 & 61.36 & 4.37 & $4.15-4.59$ \\
\hline Anterior & 102 & 38.64 & 4.45 & $4.33-4.57$ \\
\hline \multicolumn{5}{|c|}{ Tooth affected (jaw) } \\
\hline Maxilla & 151 & 57.2 & 4.46 & $4.28-4.64$ \\
\hline Mandible & 113 & 42.8 & 4.31 & $4.18-4.44$ \\
\hline
\end{tabular}

* P: 0.0052 (independent t-test)

Table 4. Dentine hypersensitivity by age, gender and tooth affected

les $(3,8,12,16,17,21,22,26)$. Only in one study men were more affected than women (18). The male/female ratios found by the clinical examination in all age groups are in agreement with the above mentioned observations. However, the reasons for difference between the two genders regarding the prevalence of $\mathrm{DH}$ are not yet clear. It has been attributed to the fact that women have better overall healthcare and oral hygiene awareness, which would make them more sensitive to $\mathrm{DH}$. It is also known that the prevalence of $\mathrm{DH}$ varies with age. Previous studies reported the peak prevalence at ages 20-29 years old (28), 30-39 years old $(3,4,28), 31-40$ years old (8), 30-39 years old $(12,13,17), 40-45$ years old (7), 40-49 years old (5) and 50-59 years old $(11,14)$. The present study showed the highest prevalence $(25.41 \%)$ occurring in the 50-59 year-old age group, consequently it is seemed to be more pertinent than other previous studies because tooth wear and periodontal disease become more common with ageing.

Declining hypersensitivity symptoms after the age of 60 may be due to the development of secondary or sclerotic dentine, and previous studies have not necessarily included large numbers of subjects over 50 years of age due to extensive tooth loss, particularly in the posterior region, or having teeth that were excluded from testing due to heavily restored teeth. The results of the present study confirm the above findings as showed that the least number of subjects reporting hypersensitivity symptoms was in the 60-69 year-old age group (DH prevalence: $12.4 \%$ ).

The mean number of sensitive teeth per patient showed a peak at 4.49 in the 30-39 year age group and then reduced slowly in the older and younger cohorts. It is also obvious that the highest mean number of sensitive teeth 
per patient occurring in the year-old age group (5.25) despite the fact that only $12.4 \%$ of these subjects report hypersensitivity symptoms. This finding could be attributed to the highest number of missing teeth of that group than the other groups.

In males the mean number of sensitive teeth per patient showed a peak at 5.77 in the 60-69 year age group, while in females the peak is shown at 4.88 in the same year age group. The findings of previous studies are not in agreement with the findings of the present study as based on different age groups. For example Rees (3) showed that the mean number of sensitive teeth per patient reached a peak at 3.7 in the 40-49 year age group and then reduced slowly in the older cohorts while for the age group 30-39 year the mean number was 3.4.

Subjects with higher (university) and lower (primary) educational level showed more sensitive teeth than those who had secondary or college education. These findings are in agreement with the findings of other studies $(4,14)$.

Similarly, subjects who belong to the I and II social class presented more sensitive teeth that those who belong to the other social classes. It is also known that DH occurs more frequently in higher social classes, who seem to brush their teeth more attentively (3).

These observations could be attributed to the fact that more educated and prosperous subjects have realized the value and importance of preventive dentistry and oral hygiene, use the available means for dental plaque control and follow a regular dental follow- up. However, previous studies have reported that gingival abrasion, recession and mechanical trauma were associated with frequency (29), tooth brushing technique (especially horizontal-scrub technique) (29).

The teeth most often affected by DH were the first premolars of both jaws followed by second premolars, canines and first molars of both jaws, according to the results of the present study due to their position in dental arch. Other studies have reported premolars and/or first molars as the most common sensitive teeth $(3-5,12,14,16,18,20$ $22,24,26,28)$ and canines $(8,16,20,28)$ while Taani and Awartani $(6,16)$ and Rees et al. (7) reported that lower incisors were one of the tooth types that were mainly affected. In the present study all the sensitive teeth also showed some degree of gingival recession. Most sensitive teeth $(85.9 \%)$ had at least $1-3.0 \mathrm{~mm}$ of gingival recession which is similar to the average recession of $2.5 \mathrm{~mm}$ reported by Addy et al. (30) in their sample of sensitive teeth.

The major stimulus that caused DH was cold drinks followed by hot drinks, tooth brushing and sour stimuli. Those observations are in agreement with findings of previous studies $(2,3,10,12,13,17,18,22,28)$. Regarding the mechanism which acts and causes DH, it is known that erosive foods (fresh fruits such as apple, citrus fruit and grape) fruit juices and beverages can remove the dentinal smear layer and increase the patency of the dentinal tubules, thereby exacerbating $\mathrm{DH}$.

Overall the general dental practioners used many different methods to treat $\mathrm{DH}$ including advice and reassurance, dietary analysis and counselling, tooth brushing modification, fluoride mouthwashes and varnish, desensitising toothpastes, applica-tion of dentine bonding agents to seal patent dentinal tubules and cervical restorations.

Regarding the role of erosive agents, it is now well established that erosive foods and drinks can remove the dentinal smear layer and increase the patency of the dentinal tubules, thereby exacerbating DH. Of these methods the most commonly prescribed was the use of desensitising toothpastes and this was often combined with a daily fluoride mouthwash or the application of a fluoride varnish in the surgery $(3,4)$.

Oral hygiene instructions including correct tooth brushing techniques as well as a regular dental follow-up could play a significant role in prevention of $\mathrm{DH}$.

In conclusion the prevalence of $\mathrm{DH}$ attending a private practice was $18.2 \%$, while the most affected teeth were the premolars, first and second and canines of both jaws and the mean number of sensitive teeth per patient showed a peak in the 30-39 year age group and then reduced slowly in the older, up to 50-59 year age group, and younger cohorts. The majority $(85.9 \%)$ of sensitive teeth had at least 1-3 mm of gingival recession while extensive gingival recession $(\geq 4 \mathrm{~mm})$ concerned $14.1 \%$ of sensitive teeth. The most common pain-initiating stimuli were the consumption of cold drinks followed by the consumption of hot drinks and tooth brushing.

\section{References}

1. Canadian Advisory Board on Dentine Hypersensitivity. Consensus-based recommendations for the diagnosis and management of dentine hypersensitivity. J Can Dent Assoc. 2003; 69: 221-6

2. Que K, Ruan J, Fan X, Liang X, Hu D. A multi-centre and crosssectional study of dentine hypersensitivity in China. J Clin Periodontol. 2010; 37: 631-7

3. Rees JS. The prevalence of dentine hypersensitivity in general dental practice in the UK. J Clin Periodontol. 2000; 27: 860-5

4. Rees JS, Addy M. A cross-sectional study of dentine hypersensitivity. J Clin Periodontol.2002; 29: 997-1003

5. Rees JS, Addy M. A cross-sectional study of buccal cervical sensitivity in UK general dental practice and a summary review of prevalence studies. Int J Dent Hyg. 2004; 2: 64-9

6. Taani SD, Awartani F. Clinical evaluation of cervical dentine sensitivity (CDS) in patients attending general dental clinics (GDC) and periodontal specialty clinics (PSC). J Clin Periodontol. 2002; 29: 118-22

7. Rees J S, Jin L J, Lam S, Kudanowska I, Vowles, R. The prevalence of dentine hypersensitivity in a hospital clinic population in Hong Kong. J Dent. 2003;31:453-61

8. Udoye CI. Pattern and distribution of cervical dentine hypersensitivity in a Nigerian tertiary hospital. Odontostomatol Trop. 2006 ; 29: 19-22

9. Chabanski MB, Gillam DG, Bulman JS, Newman HN. Clinical evaluation of cervical dentine sensitivity in a population of patients referred to a specialist periodontology department: a pilot 
study. J Oral Rehabil. 1997; 24: 666-72

10. Irwin C R, McCusker P. Prevalence of dentine hypersensitivity in a general dental population. J Irish Dent Assoc. 1997; 43: 7-9

11. Liu HS, Lan WH, Hsieh CC. Prevalence and distribution of cervical dentine hypersensitivity in a population in Taipei, Taiwan. $\mathrm{J}$ Endod. 1998; 24: 45-7

12. Amarasena N, Spencer J, Ou Y, Brennan D. Dentine hypersensitivity in a private practice population in Australia. J Oral Rehabil. 2011; 38: 52-60

13. Amarasena N, Spencer J, Ou Y, Brennan D. Dentine hypersensitivity-Australian dentists' perspective. Aust Dent J. 2010; 55: 181-7

14. Kehua Q, Yingying F, Hong S, Menghong W, Deyu H, Xu F. A cross-sectional study of dentine hypersensitivity in China. Int Dent J. 2009; 59: 376-80

15. Gillam DG, Bulman JS, Eijkman MA, Newman HN. Dentists' perceptions of dentine hypersensitivity and knowledge of its treatment. J Oral Rehabil.2002; 29: 219-25

16. Taani DQ, Awartani F. Prevalence and distribution of dentine hypersensitivity and plaque in a dental hospital population. Quintessence Int. 2001; 32: 372-6

17. Gillam DG, Seo HS, Bulman JS, Newman HN. Perceptions of dentine hypersensitivity in a general practice population. J Oral Rehabil. 1999; 26: 710-4

18. Bamise CT, Olusile AO, Oginni AO, Dosumu OO. The prevalence of dentine hypersensitivity among adult patients attending a Nigerian teaching hospital. Oral Health Prev Dent. 2007; 5: 49-53

19. Banoczy J. Dentine hypersensitivity and its significance in dental practice. Fogorv Sv. 2002; 95: 223-8

20. Miglani S, Aggarwal V, Ahuja B. Dentine hypersensitivity: Recent trends in management. J Conserv Dent. 2010; 13: 218-24

21. Ye W, Wang GY, Ly J, Feng XP. The epidemiology of dentine hypersensitivity among adults in Shanghai municipality. Shanghai Kou Qiang Yi Xue. 2009;18:247-50

22. Tan CS, Hu DY, Fan X, Li X, Que KH. Epidemiological survey of dentine hypersensitivity of young people in Chengdu City. Hua Xi, Kou Qiang Yi Xue Za Zhi 2009; 27: 394-6

23. Bartold PM. Dentinal hypersensitivity: a review. Aust Dent J. 2006; 51:212-8

24. Walters PA. Dentinal hypersensitivity: a review. J Contemp Dent Pract. 2005; 2:107-17

25. Chabanski MB, Gillam DG, Bulman JS, Newman HN. Prevalence of cervical dentine sensitivity in a population of patients referred to a specialist periodontology department. J Clin Periodontol. 1996; 23: 989-92

26. Dababneh R H, Khouri A T, Addy M. Dentine hypersensitivity an enigma? A review of terminology, epidemiology, mechanisms, aetiology and management. Brit Dent J. 1999; 187: 606-11

27. Bradnock G, White DA, Nutall WM, Morris AJ, Treasure ET, Pine CM. Dental attitudes and behaviour in1998 and implications for the future. Br Dent Jour.2001;190: 229-32

28. Gillam DG, Aris A, Bulman JS, Newman HN, Ley F. Dentine hypersensitivity in subjects recruited for clinical trials: clinical evaluation, prevalence and intra-oral distribution. J Oral Rehabil. 2002; 29: 226-31

29. Akgül HM, Akgül N, Karaoglanogu S, Ozdabak N.A survey of the correspondence between abrasions and tooth brushing habits in Erzurum, Turkey. Int Dent J. 2003; 33: 491-5

30. Addy M, Mostafa P, Newcombe R G. Dentine hypersensitivity: the distribution of recession, sensitivity and plaque. J Dent. 1987 ;15: 242-8 